\title{
On the Occurrence of Centrospheres in Pellia epiphylla, Nees.
}

\author{
BY \\ J. BRETLAND FARMER, M.A., \\ Assistant Professor of Biology, Royal College of Sciesuce, Lonion, \\ AND \\ JESSE REEVES, \\ Marshall Sicholar, Royal College of Science, London. \\ With Plate XIV.
}

T $\mathrm{T}$ is a well-known fact that the spores of Pellia epiphylla, 1 like those of some other Liverworts, germinate while still enclosed in the sporogonium, and that long before the wall of the capsule ruptures, the spores have become multicellular bodies. The spores of Pellia, when in this condition, afford excellent material for the study of the karyokinesis, owing to the large size of their nucleus and the relatively small number of its chromosomes. But the special interest which attaches to them lies in the singular degree of clearness with which the attraction-spheres (or centrospheres) are differentiated, and in the facility which is thereby afforded for the study of these structures in their relation to the process of nuclear division.

For the purpose of this investigation, fruiting plants of Pellia were gathered during the winter, and were preserved [Annals of Botany, Vol. VIII. No. XXX. June, 1894.]

Q 2 


\section{2 io Farmer and Reeves.-On the Occurrence of}

in strong spirit, which, so far as the present plant is concerned, we find to give results in no way inferior to those obtained by the use of absolute alcohol. The sporogonia, with their contained spores, were embedded in paraffin, and cut with the microtome in the usual way. For staining purposes we employed a considerable variety of reagents, but we obtained the best results after using the following double stains ;--gentian violet and orange G., gentian violet and eosin, aniline blue and acid fuchsin. In every instance we used the stains successively, and on the whole the second of the above combinations gave the most satisfactory preparations. It should be noted, however, that in dealing with these structures, we find it by no means follows that a given treatment which may answer admirably for one plant, will succeed equally well in the case of another, and in this we are in accord with the experience of those who have devoted attention to animal centrosomes. The reason of the difference is probably to be sought in the slight chemical or physical differences which exist between the protoplasmic structures in different organisms, but our knowledge of the intimate constitution of these bodies is at present so scanty that we are compelled to have recourse to empirical experiment, in order to determine which reagent may be the best to use in each particular case.

The spores of Pellia epiphylla are crowded with starchgrains, and contain, as has been already said, a nucleus of considerable size, in which the chromatin occurs in a condition of aggregation during the resting stage. The nuclear membrane is extremely sharply defined, and within the body of the nucleus a nucleolus may be seen, though it is not always easy to distinguish.

When nuclear division is about to take place, two structures of a minute size appear on the outside of, and in contact with, the nuclear wall, and from them beautiful radiations extend. These bodies, or centrospheres, are commonly seen to be diametrically opposite to each other in position, for we have not succeeded in demonstrating them in the perfectly resting 
cells, nor have we been able to ascertain the existence of any definite particle within them which would indicate the presence of a centrosome; it is true that in some instances such a point could be distinguished, but we do not attach much importance to it, since in the great majority of centrospheres it completely eluded recognition. And, although the radiation in the cytoplasm may readily be traced into the protoplasmic corpuscle, we prefer for the present, at any rate, to term this a centrosphere rather than a centrosome, since it appears probable that the corpuscle is really equivalent to something more than the centrosome alone.

The cytoplasmic radiations are exceedingly clear in this plant, and especially well seen when regarded from above, i.e. in the direction of the polar axis, and they are then seen to spread out in a star-like manner from a common centre (PI. XIV, Fig. 5).

Presently the centrospheres assume such a relation to the nucleus that one can hardly resist interpreting it as a pulling strain. The nucleus becomes more and more drawn out into an elliptical shape, and at the somewhat pointed ends the c $€ n t r o s p h e r e s$ are respectively situated (Pl. XIV, Fig. 2). The chromatin now becomes distributed in the form of a narrow equatorial band lying just within the nuclear membrane which still persists, and indeed, in this equatorial region, exhibits, if possible, a sharper outline than before. The chromatin gradually becomes more definitely fibrillar, and finally appears in the form of eight chromosomes, which can easily be counted when the equatorial nuclear plate is formed. Meanwhile the rest of the nucleus is entirely free from staining-substance, and its ends become more and more drawn out, whilst its wall becomes pari passu, much thinner, except just outside the chromatic band mentioned above. The cytoplasmic radiations increase in number and distinctness, and extend over the attenuated nuclear wall. Concomitantly with the final disappearance of the latter, the achromatic spindle is differentiated, and rapidly becomes very prominent, owing to its growing capacity for taking stains. It is hardly possible, 


\section{Farmer and Reeves.-On the Occurrence of}

in a case like the present, to escape the conviction that there exists a close relation between the spindle and the nuclear wall, over which the radiations have been extending. The centrospheres frequently become difficult to distinguish at this stage, and the polar radiations are often hardly visible. The achromatic spindle apparently ends sharply at a point, but we have convinced ourselves, as the result of a careful examination of a somewhat extensive series of preparations, that the spindle does not, as a matter of fact, terminate in the general cytoplasm, but that its two apices are respectively occupied by two (sometimes only one) corpuscles, and we believe we are justified in regarding these as the representatives of the centrospheres, although they do not exhibit a degree of clearness such as that described by Schottländer ${ }^{1}$ for the antherozoid-mother-cells of Marchantia. On the other hand, the radiations are obvious here, while Schottländer states that with one exception these did not occur in the plants investigated by him.

After the separation of the chromosomes from the equator to form the daughter nuclei, a beautiful cell-plate is formed across the achromatic spindle, by local thickening of the 'Verbindungsfäden,' and it then extends centrifugally, finally meeting the peripheral wall of the cell, exactly as in the higher plants.

During the reformation of the daughter-nuclei the radiations above spoken of at first become again more distinct, but they finally die away altogether, and in the completely resting nuclei they are entirely wanting. When this stage is reached we do not succeed in definitely detecting the presence of a centrosphere or centrosome, and we do not regard it as probable that these structures retain their individuality within the cytoplasm in the case of Pellia. It is easy enough to find bodies of minute size, surrounded by their diffraction areas, but it would be pure empiricism to fix on any one or

1 Schottländer, Beitr. z. Kentniss d. Zellkerne u. d. Sex. Zell. b. d. Kryptogamen. Cohn, Beitr. z. Biol. d. Pflanzen, Hd. VI, 1892. 
two of these and call them centrosomes. Nor is this surprising, since even in many favourable cases of cell-division in animals it is by no means clear as to what is the fate of these bodies, whether they become diffused in the general cytoplasm, or whether they become retracted into the nucleus. If they really do persist in Pellia, the latter view seems perhaps the least improbable, owing to the intimate relations in space which always obtain between nucleus and centrospheres in this plant, and which have been already alluded to.

The processes of division, as described above, are subject to some deviations. In a few instances, one or both of the centrospheres, with their attendant radiations, were seen in positions apparently remote from the nuclear wall, during the earliest phases of division. This appearance proved, however, on examination, to be illusory, and to be due to the fact that the centrospheres had not taken up diametrically opposite positions outside the nucleus, so that when this body begins to be drawn out, it assumes an asymmetrical, or more correctly stated, a bilaterally symmetrical shape (Figs. 3,4 ). If, then, the distortion is very great, and the observer happens to regard the nucleus from the convex side, it is easy to overlook the oblique prolongations of the membrane, and hence the asters appear to be situated in the cytoplasm and away from the nucleus (Fig. 4). It is worth notice that in all asymmetrical nuclei of this nature the belt of chromatin is always broader on the outer than on the inner curve.

When the asymmetrical condition becomes very strongly marked, a third centrosphere, often feebler than the other two, may be occasionally observed. We have not seen any simultaneous division of the nucleus into three daughter nuclei, although occasionally the arrangement of the chromatin is such as to suggest that this might occur; as an alternative, it is possible that the weakest centrosphere ultimately becomes absorbed by one of the other two, and this we regard as the most likely explanation of the fact that even in preparations which exhibited three centrospheres, we never saw three spindles, or any such arrangement of cell-walls in completely 


\section{Farmer and Reeves.-On Pellia epiphylla, Nees.}

divided cells as would support the view of a simultaneous division having taken place.

It is impossible to avoid being struck by the differences existing between the processes accompanying nuclear division in the spores of this Liverwort, and those obtaining in certain other plants. We reserve, however, the consideration of these for a future paper, contenting ourselves in this place with a description of the facts as they may be observed in Pellia epiphylla.

\section{EXPLANATION OF FIGURES IN PLATE XIV.}

Illostrating Messrs. Farmer and Reeves' paper on the Occurrence of Centrospheres in Pellia epiphylla, Nees.

All the figures refer to Pellia, and are drawn from spores germinating within the sporogonium.

Fig. I. Mature spore with the nucleus in the resting condition.

Fig. 2. Nucleus becoming elliptical. The centrospheres at the two poles. The chromatin aggregated in an equatorial belt.

Fig. 3 and 4. Nucleus assaming an asymmetrical shape. The centrospheres not diametrically opposed.

Fig. 5. A polar view of the centrosphere and the radiations.

Fig. 6 and 7. Nacleus in which chromatin fibrils are becoming clear.

Fig. 8. Later stage of karyokinesis.

Fig. 9. Chromosomes, eight in number, seen in polar view.

Fig. 1o. Daughter-nuclei in process of reformation. A marked cell plate.

Fig. II. Spore which has divided into three cells, in each of which stages of nuclear division are shown. 
Fig.

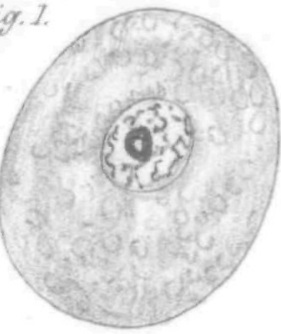

Fig. 4.

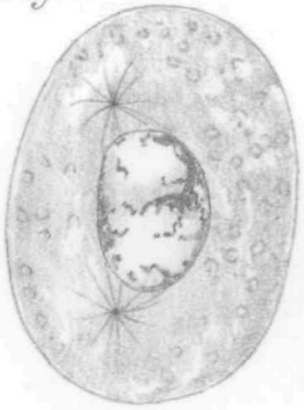

Fig. 5 .

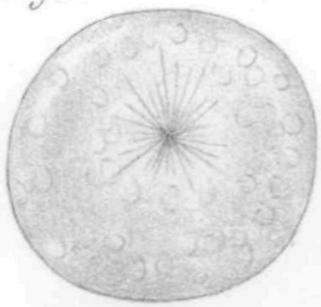

Fig. 8 .

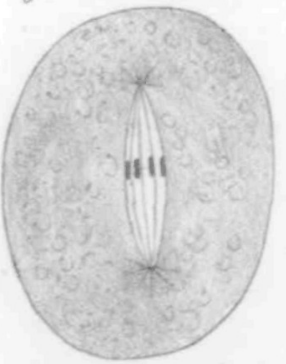

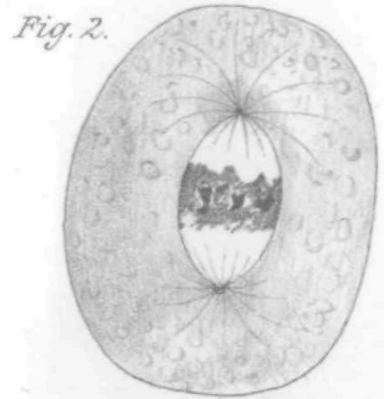

Fig.3.

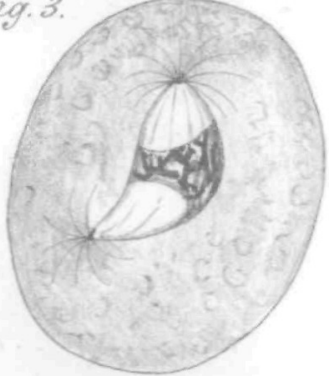

Fig. 6

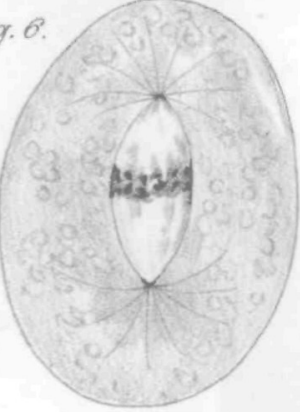

Fig. \%.

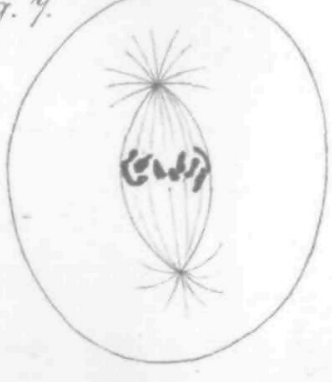

Fig. II.

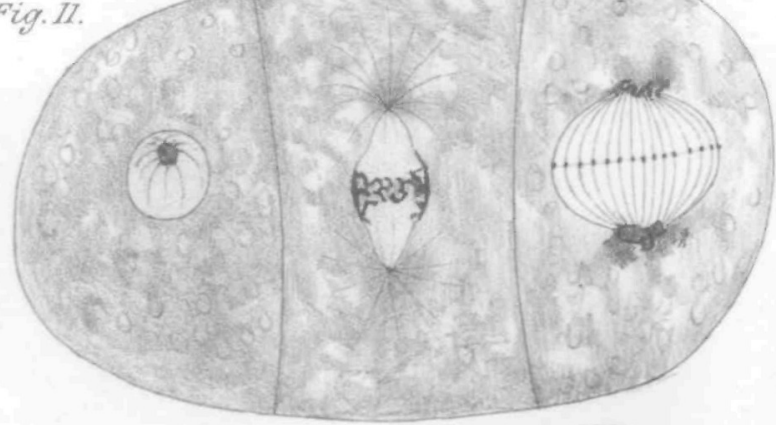

Fig.9.
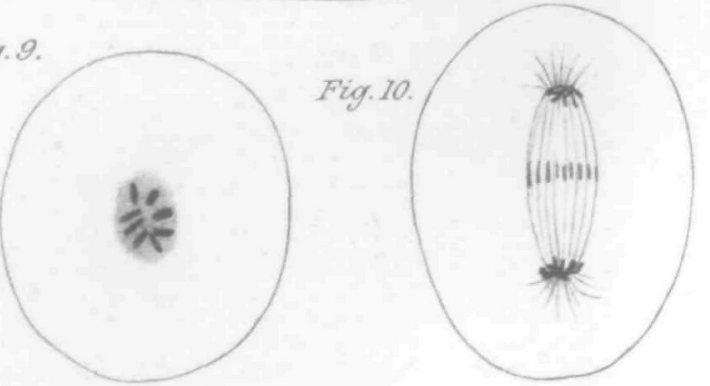

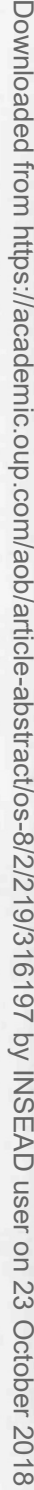

University Press, 0xford. FARMER. - PELLIA EPIPHYLLA. 